\title{
Numerical Investigation of Thermal Stability of Catalyst Granules with Internal Heat Generation in a Random Temperature Field
}

\author{
Igor Derevich, Daria Galdina \\ Department of Applied Mathematics \\ Moscow State University by N.E. Bauman (MSTU) \\ Russian Federation \\ DerevichIgor@gmail.com, galdaria@mail.ru
}

Received: February 15, 2021. Revised: March 8, 2021. Accepted: March 15, 2021. Published: March 29, 2021.

\begin{abstract}
Method for numerical simulation of temperature of granules with internal heat release in a medium with random temperature fluctuations it is proposed. The method utilized solution of a system of ordinary stochastic differential equations describing temperature fluctuations of surrounding and granules. Autocorrelation function of temperature fluctuations has a finite decay time. The suggested method is verified by the comparison with exact analytical results. Random temperature behavior of a granule with internal heat release qualitatively differs from the results obtained in the deterministic approach. Mean first passage time of granules temperature intersects critical temperature is estimated at different regime parameters.
\end{abstract}

Keywords-stochastic ordinary differential equation; autocorrelation function; heat explosion; Semenov's diagram; temperature fluctuations

\section{INTRODUCTION}

The catalytic synthesis processes generally accompanied by heat release. Synthesis of heavy hydrocarbons in the FischerTropsch process (GTL technology) is associated with essential heat generation [1]. GTL technology can solve a number of environmental and economic problems.

In the Technological Institute for Superhard and Novel Carbon Materials (Troitsk, Russian Federation) is developed industrial reactor with capacity $5000 \mathrm{Nm}^{3} / \mathrm{h}$ of synthesis gas with production $500 \mathrm{~kg} / \mathrm{h}$ stabilized liquid hydrocarbons. The reactor used fixed bed of catalyst granules.

Exothermic heat of reaction is transferred from the volume of catalytic granules to the boundary of the granules. At the boundary heat is removed to the liquid products of the synthesis. Exceeding heat generation over heat transfer leads to uncontrolled growth temperature - thermal explosion. Loss of thermal stability of catalyst granules can lead to thermal explosion of the reactor. Therefore, investigation critical conditions of thermal explosion are important practical problem.
Conditions leading to thermal explosion in deterministic situation have been well studied [2-6]. There is a critical temperature, the excess of which causes a significant increase in temperature of granules. The situation is qualitatively different when the temperature of the environment is a random process. In this case there is always a non-zero probability exists a temperature fluctuation, the magnitude of which exceeds a critical value, which may lead to loss of thermal stability. Study of the effect of random noise on the behavior of systems with explosive behavior dedicated, for example, [711]. The results of this study can also be applicable in modeling of ignition conditions of dispersed fuel in aircraft and rocket engines, power stations. Main trends obtained in the paper are helpful for estimation probability of thermal explosion in storages and transportation lines of dispersed combustible materials.

Investigation effect of noise on behavior of systems with explosive behavior in papers [7-11] was carried out in the framework of probability density function approach [12]. This approach requires the use of modern methods of stochastic processes and functional analysis and yields results which have practical importance. However, the method of the probability density function does not take into account the some important details of the detailed chemical kinetics. In this situation, appropriate to use the methods of direct numerical modeling of temperature dynamics on the basis of solutions of stochastic ordinary differential equations [13-17].

In this paper we propose a method for direct numerical simulation of random temperature of granules with internal heat generation and with temperature fluctuations in the surronding. We construct temperature fluctuations with internal temporal structure. The autocorrelation function of themperature fluctuation of the fluid has finite decay time. This approach can be used in future for modeling stochastic behavior not only temperature, but reactant concentration inside the granules with detailed kinetics. Verification of the proposed algorithm is based on a comparison with analytical solutions. We Illustrate the various scenarios the loss of 
thermal stability of catalytic granules. Calculations results of the average waiting time of thermal explosion are presented.

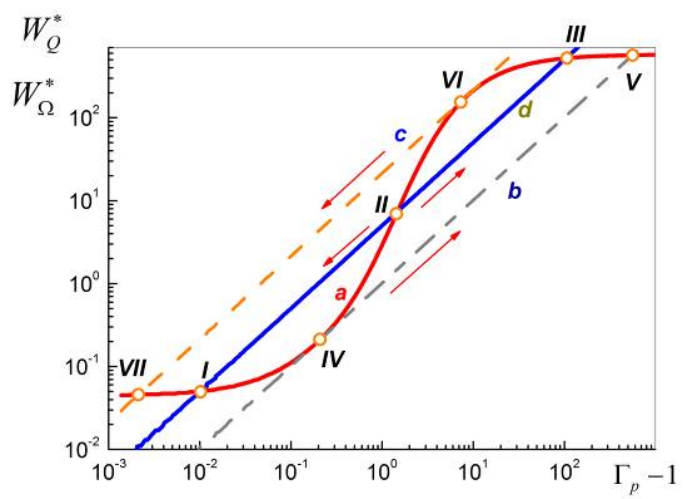

Fig. 1. Semenov's diagram.

\section{EQUATION FOR TEMPERATURE OF A GRANULE WITH INTERNAL HEAT RELEASE. SEMENOV'S DIAGRAM}

\section{A. Equation for temperature of the catalytic granule}

We investigate spherical granule with diameter $d_{p}$, which is placed in liquid products with temperature $\Theta_{f}$. Thermal effect of exothermal reaction inside the granule is $Q$. Rate of chemical reactions is modeled as Arrhenius law with activation energy $E$. Heat transfer coefficient is $\alpha$. Equation for the volume-averaged temperature of the granule $\Theta_{p}$ has the following form

$$
m_{p} c_{p} \frac{\mathrm{d} \Theta_{p}}{\mathrm{~d} t}=\alpha S_{p}\left(\Theta_{f}-\Theta_{p}\right)+V_{p} Q A \mathrm{e}^{-\frac{E}{R^{\circ} \Theta_{p}}} .
$$

Here $m_{p}$ is mass of the granule; $S_{p}=\pi d_{p}^{2}$ area of granule surface; $V_{p}=\pi d_{p}^{3} / 6$ is volume of the granule; $A$ is the frequency factor; $R^{\circ}$ is universal gas constant.

The equation for the granule temperature can be rewritten in the relaxation form

$$
\frac{\mathrm{d} \Theta_{p}}{\mathrm{~d} t}=\frac{\Theta_{f}-\Theta_{p}}{\tau_{\Theta}}+\frac{Q A}{\rho_{p} c_{p}} \mathrm{e}^{-\frac{E}{R^{\circ} \Theta_{p}}},
$$

where $\tau_{\Theta}=d_{p} c_{p} / \alpha_{p}$ is temperature relaxation time of the granule.

Temperature of liquid is given as

$$
\Theta_{f}(t)=\left\langle\Theta_{f}\right\rangle+\theta_{f}(t)
$$

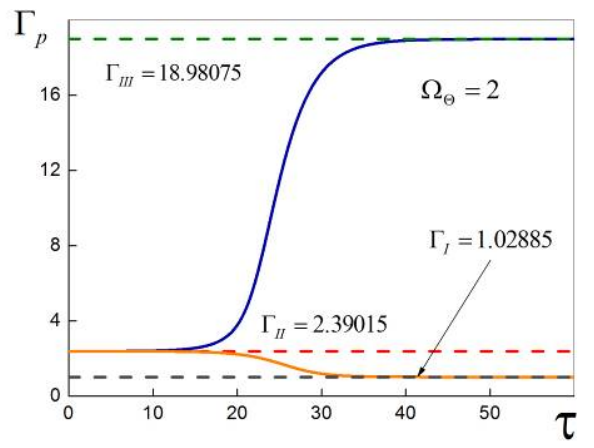

Fig. 2. Behavior of temperature close to second root on Semenov's diagram

Where $\left\langle\Theta_{f}\right\rangle$ is averaged temperature of the fluid; $\theta_{f}(t)$ is temperature fluctuations, $\left\langle\theta_{f}(t)\right\rangle=0$, angular brackets denote the results of averaging over an ensemble of random process of fluid temperature.

Equation (1) in dimensionless variables has the form

$$
\frac{\mathrm{d} \Gamma_{p}}{\mathrm{~d} \tau}=\frac{\left(1+\gamma_{f}\right)-\Gamma_{p}}{\Omega_{\Theta}}+Q^{*} \mathrm{e}^{-\frac{E^{*}}{\Gamma_{p}}},
$$

Here $\Gamma_{p}=\Theta_{p} /\left\langle\Theta_{f}\right\rangle$ is dimensionless temperature of the granule; $\tau=t / T_{E}$ is dimensionless time; $T_{E}$ is integral time scale of fluid temperature autocorrelation function; $\gamma_{f}=\theta_{f} /\left\langle\Theta_{f}\right\rangle$ is a dimensionless temperature fluctuation of liquid phase; $E^{*}=E / R^{\circ}\left\langle\Theta_{f}\right\rangle$ is dimensionless energy activation; $Q^{*}=Q A /\left(\rho_{p} c_{p}\left\langle\Theta_{f}\right\rangle\right)$ is dimensionless heat of exothermal reaction; $\Omega_{\Theta}$ is parameter of thermal inertial of the granule.

\section{B. Semenov's diagram}

Based on the analysis of Semenov's critical diagram we show the existence of critical temperature. Infinitely small excess above the critical temperature leads to uncontrolled increase in temperature of the granule - thermal explosion.

Analysis of Semenov's diagram is provided for steady-state temperature of the carrier medium. Looking for a stationary temperature of the granule

$$
\frac{\mathrm{d} \Gamma_{p}}{\mathrm{~d} \tau}=0, \frac{\Gamma_{p}-1}{\Omega_{\Theta}}=Q^{*} \mathrm{e}^{-\frac{E^{*}}{\Gamma_{p}}} .
$$

We introduce dimension less power of heat transfer to the liquid phase $W_{\Omega}^{*}=\left(\Gamma_{p}-1\right) / \Omega_{\Theta}$ and dimensionless power of heat release $W_{Q}^{*}=Q^{*} \exp \left(-E^{*} / \Gamma_{p}\right)$. 
Figure 1 represent Semenov's diagram. It is evident that there is a region with three fixed temperatures of the granule. This region of three roots of equation (3) is bounded by the tangential lines, whose position is determined by the values of thermal relaxation parameter of the granule.

At the tangential lines $\mathrm{b}$ and $\mathrm{c}$ in Fig. 1 the temperature of the granule returns to a steady state with low or high temperatures, respectively. To study the types of stationary temperature we performed numerical integration of the nonlinear equation (2) without taking into account fluctuations in the temperature of the medium. Figure 2 illustrates the dynamics of change of temperature granules, if the initial temperature is close to the second root on the Semenov. It can be seen that an infinitely small temperature above $\Gamma_{I I}$ give a loss of thermal stability of the granule.

If the initial temperature of the granule is infinitively less than the value $\Gamma_{I I}$, the temperature of the granules proceeds to low temperatures close to ambient temperature. The second root at the Semenov's diagram may be regarded as critical value $\Gamma_{c r}=\Gamma_{I I}$.

\section{AUTOCORRELATION FUNCTION OF TEMPERATURE FLUCTUATIONS. EXACT RESULTS}

In this section, we obtain some exact results for comparison with data of numerical simulation. Exact solutions exist for linear equations. We consider the equation for the fluctuations of temperature granules (1) without a chemical heat source

$$
\frac{\mathrm{d} \theta_{p}(t)}{\mathrm{d} t}=\frac{\theta_{f}(t)-\theta_{p}(t)}{\tau_{\Theta}} .
$$

Temperature fluctuations of fluid $\theta_{f}(t)$ is statistically stationary random process with correlation

$$
\left\langle\theta_{f}\left(t^{\prime}\right) \theta_{f}\left(t^{\prime \prime}\right)\right\rangle=\left\langle\theta_{f}^{2}\right\rangle \Psi_{f}\left(t^{\prime}-t^{\prime \prime}\right) .
$$

We use the relationship between the autocorrelation function and its spectrum

$$
\begin{gathered}
\Psi_{f}(t)=\frac{1}{(2 \pi)} \int_{-\infty}^{\infty} e^{-i \omega t} \Psi_{f}(\omega) d \omega, \\
\Psi_{f}(\omega)=\int_{-\infty}^{\infty} e^{i \omega s} \Psi_{f}(s) d s .
\end{gathered}
$$

Solution of equation (4) has the form

$$
\theta_{p}(t)=\frac{1}{\tau_{\Theta}} \int_{0}^{t} e^{-\frac{t-s}{\tau_{\Theta}}} \theta_{f}(s) d s
$$

Autocorrelation function of temperature fluctuations of the granule is written as

$$
\left\langle\theta_{p}\left(t^{\prime}\right) \theta_{p}\left(t^{\prime \prime}\right)\right\rangle=\left\langle\theta_{p}^{2}\right\rangle \Psi_{p}\left(t^{\prime}-t^{\prime \prime}\right)
$$

$$
=\frac{1}{\tau_{\Theta}} \int_{0}^{t^{\prime}} d s^{\prime} e^{-\frac{t^{\prime}-s^{\prime}}{\tau_{\Theta}}} \frac{1}{\tau_{\Theta}} \int_{0}^{t^{\prime \prime}} d s^{\prime \prime} e^{-\frac{t^{\prime \prime}-s^{\prime \prime}}{\tau_{\Theta}}}\left\langle\theta_{f}\left(s^{\prime}\right) \theta_{f}\left(s^{\prime \prime}\right)\right\rangle
$$

With the help of spectrum of the fluid temperature autocorrelation function (5) and (6) we write down expression for granule autocorrelation

$$
\left\langle\theta_{p}^{2}\right\rangle \Psi_{p}(t)=\frac{\left\langle\theta_{f}^{2}\right\rangle}{2 \pi} \int_{-\infty}^{\infty} e^{-i \omega t} \frac{\psi_{f}(\omega)}{1+\left(\omega \tau_{\Theta}\right)^{2}} d \omega .
$$

Square of dispersion of the granule temperature fluctuations is follows from expression (8)

$$
\left\langle\theta_{p}^{2}\right\rangle=\frac{\left\langle\theta_{f}^{2}\right\rangle}{2 \pi} \int_{-\infty}^{\infty} \frac{\psi_{f}(\omega)}{1+\left(\omega \tau_{\Theta}\right)^{2}} d \omega .
$$

Let us consider two special cases of the autocorrelation function of the temperature fluctuations of the fluid.

Random process $\theta_{f}(t)$ - is delta-correlated in time random process. The autocorrelation function (5) has the form

$$
\Psi_{f}\left(t^{\prime}-t^{\prime \prime}\right)=2 \tau_{\circ} \delta\left(t^{\prime}-t^{\prime \prime}\right)
$$

Here $\tau_{\circ}$ is integral time scale

$$
\int_{0}^{\infty} \Psi_{f}(s) d s=2 \tau_{\circ} \int_{0}^{\infty} \delta(s) d s=\tau_{\circ} .
$$

Spectrum of autocorrelation function (9) is found from expression (7)

$$
\psi_{f}(\omega)=2 \tau_{\circ} \int_{-\infty}^{\infty} e^{i \omega s} \delta(s) d s=2 \tau_{\circ} .
$$

Substitution expression for the spectrum into formula (8) leads to autocorrelation function of the granule temperature fluctuations

$$
\left\langle\theta_{p}^{2}\right\rangle \Psi_{p}(t)=\left\langle\theta_{f}^{2}\right\rangle \frac{\tau_{\circ}}{\tau_{\Theta}} e^{-\frac{t}{\tau_{\Theta}}} .
$$

Intensity of temperature fluctuations and autocorrelation function of granule are

$$
\left\langle\theta_{p}^{2}\right\rangle=\frac{\tau_{\circ}}{\tau_{p}}\left\langle\theta_{f}^{2}\right\rangle \ll\left\langle\theta_{f}^{2}\right\rangle, \Psi_{p}(t)=e^{-\frac{t}{\tau_{\Theta}}} .
$$

Delta-correlation approach is correct for granules with high thermal inertia. Autocorrelation function of the granule temperature fluctuations has exponential form with integral time scale equal the granule relaxation time.

Second approach is exponential approximation of $\theta_{f}(t)$ fluid temperature autocorrelation function

$$
\Psi_{f}(t)=e^{-\frac{t}{T_{E}}}
$$




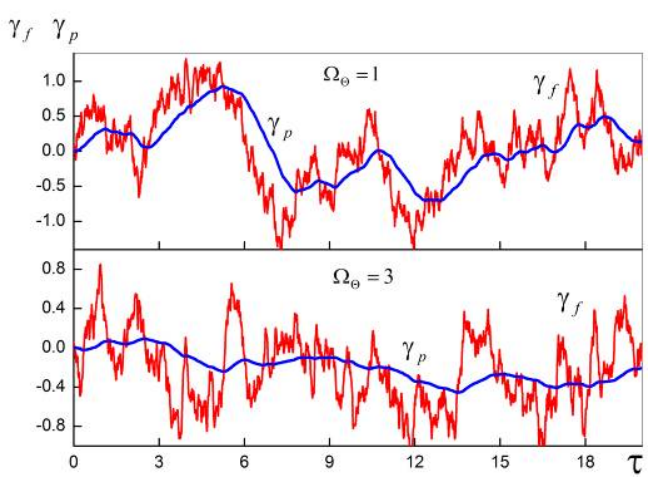

Fig. 3. Example of random realizations of ambient temperature and the granules

Spectrum of the autocorrelation function follows from formula (7)

$$
\psi_{f}(\omega)=\int_{-\infty}^{\infty} e^{i \omega t-\frac{|t|}{T_{E}}} d t=\frac{2 T_{E}}{1+\left(\omega T_{E}\right)^{2}} .
$$

Correlation of the granule temperature fluctuation is obtained from formula (8)

$$
\left\langle\theta_{p}^{2}\right\rangle \Psi_{p}(t)=\frac{\left\langle\theta_{f}^{2}\right\rangle}{2 \pi} \int_{-\infty}^{\infty} \frac{2 T_{E} e^{-i \omega t}}{\left[1+\left(\omega \tau_{\Theta}\right)^{2}\right]\left[1+\left(\omega T_{E}\right)^{2}\right]} d \omega
$$

Calculation under theory of functions with complex variables leads to the result

$$
\left\langle\theta_{p}^{2}\right\rangle \Psi_{p}(t)=\left\langle\theta_{f}^{2}\right\rangle \frac{e^{-\frac{t}{T_{E}}}-\left(\tau_{\Theta} / T_{E}\right) e^{-\frac{t}{\tau_{\Theta}}}}{1-\left(\tau_{\Theta} / T_{E}\right)^{2}} .
$$

Square of dispersion of the granule temperature fluctuations is follows from equation (11) at $t=0$

$$
\left\langle\theta_{p}^{2}\right\rangle=\frac{\left\langle\theta_{f}^{2}\right\rangle}{1+\left(\tau_{\Theta} / T_{E}\right)} .
$$

Autocorrelation function of the granule temperature fluctuation also follows from equation (11)

$$
\Psi_{p}(t)=\frac{e^{-\frac{t}{T_{E}}}-\left(\tau_{\Theta} / T_{E}\right) e^{-\frac{t}{\tau_{\Theta}}}}{1-\left(\tau_{\Theta} / T_{E}\right)} .
$$

Integral time scale of the granule temperature fluctuation is

$$
T_{\Theta}=\int_{0}^{\infty} \Psi_{p}(t) d t=T_{E}+\tau_{\Theta} .
$$

It is seen that there are two types of granules. Granules with small thermal inertia with granules relaxation times much smaller than integral time scale of temperature autocorrelation function of fluid $\tau_{\Theta} \ll T_{E}$. In that case dispersion of temperature fluctuations of granules and fluid is close $\left\langle\theta_{p}^{2}\right\rangle \approx\left\langle\theta_{f}^{2}\right\rangle$, and integral time scale of granules temperature fluctuations is $T_{\Theta} \approx T_{E}$. For granule with high thermal inertia $\tau_{\Theta} \gg T_{E}$ dispersion of granules temperature fluctuations is less then fluid $\left\langle\theta_{p}^{2}\right\rangle \approx\left(T_{E} / \tau_{\Theta}\right)\left\langle\theta_{f}^{2}\right\rangle$. Integral time scale of granule temperature fluctuations is close to temperature relaxation time $T_{\Theta} \approx \tau_{\Theta}$, and granules autocorrelation function decays as $\Psi_{p}(t) \approx \exp \left(-t / \tau_{\Theta}\right)$.

Obtained exact results used for testing numerical algorithm of simulation temperature of granules in a random temperature of fluid.

\section{SYSTEM OF STOCHASTIC DIFFERENTIAL EQUATIONS}

Analytical results show that modeling autocorrelation function with finite relaxation time is possible only on the base of stochastic ordinary equations.

Write down system of differential equations for temperature fluctuations of fluid and the granule with heat release

$$
\begin{gathered}
\frac{\mathrm{d} \gamma_{f}(\tau)}{\mathrm{d} \tau}=\eta(\tau)-\gamma_{f}(\tau) \\
\frac{\mathrm{d} \Gamma_{p}(t)}{\mathrm{d} \tau}=\frac{\left(1+\gamma_{f}(t)\right)-\Gamma_{p}(t)}{\Omega_{\Theta}}+Q^{*} \mathrm{e}^{-\frac{E^{*}}{\Gamma_{p}(t)}} .
\end{gathered}
$$

Here $\eta(t)$ - is seeded Gaussian random process with deltacorrelated function $\left\langle\eta\left(t^{\prime}\right) \eta\left(t^{\prime \prime}\right)\right\rangle=2 \tau_{\circ}\left\langle\eta^{2}\right\rangle \delta\left(t^{\prime}-t^{\prime \prime}\right)$.

Integration of the system of equations (14) and (15) is carried out by explicit Euler method

$$
\begin{gathered}
\gamma_{f}^{(n+1)}=\gamma_{f}^{(n+1)}+\Delta \tau\left\{\eta^{(n)}-\gamma_{f}^{(n)}\right\}, \\
\Gamma_{p}^{(n+1)}=\Gamma_{p}^{(n)}+\Delta \tau\left\{\frac{\left(1+\gamma_{f}^{(n+1)}\right)-\Gamma_{p}^{(n)}}{\Omega_{\Theta}}+Q^{*} \mathrm{e}^{-\frac{E^{*}}{\Gamma_{p}^{(n)}}}\right\} .
\end{gathered}
$$

Here $n-$ is the number of temporary steps; random increment of seeded process is modeled as

$$
\Delta \tau \eta^{(n)}=\xi^{(n)} \sqrt{2\left\langle\eta^{2}\right\rangle \tau_{\circ} \Delta \tau}
$$

where $\xi^{(n)}$ - is random realization of the normalized Gaussian process (white noise) with zero mean and unit dispersion.

Figure 3 illustrates the effect of thermal inertia of the granules on the character of temperature fluctuations without 
heat source. It can be seen that increasing the thermal inertia reduces the amplitude of temperature fluctuations of the granule.

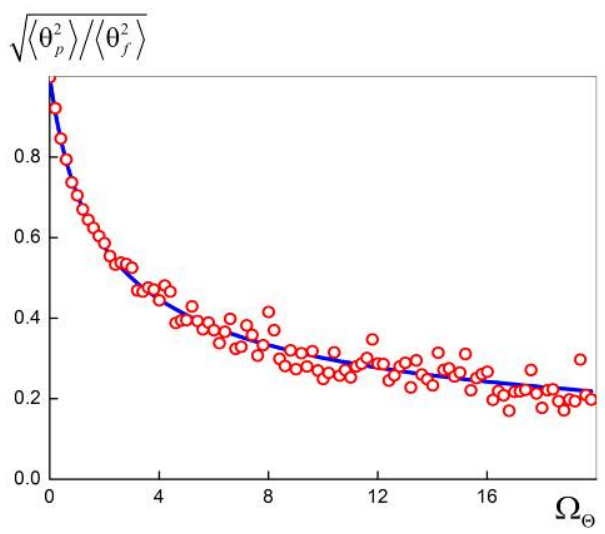

Fig. 4. The ratio dispersions of temperature fluctuations of granules to fluid: points are simulation results; curve is the formula (12).

Figure 4 shows influence of thermal inertia of granules on dispersion of temperature fluctuations. The increasing thermal inertia decreases the intensity of temperature fluctuations of granules. From the Figure 4 is also evident a satisfactory agreement between the results of calculations by the exact formula (12) and numerical data obtained by averaging random realizations of temperature.

Autocorrelation function of the granule temperature fluctuations is shown in Figure 5. It can be seen that the results of numerical simulations satisfactory agree with exact results obtained by the formula (13). The growth of thermal inertia increases the damping region of the autocorrelation function of the granules.

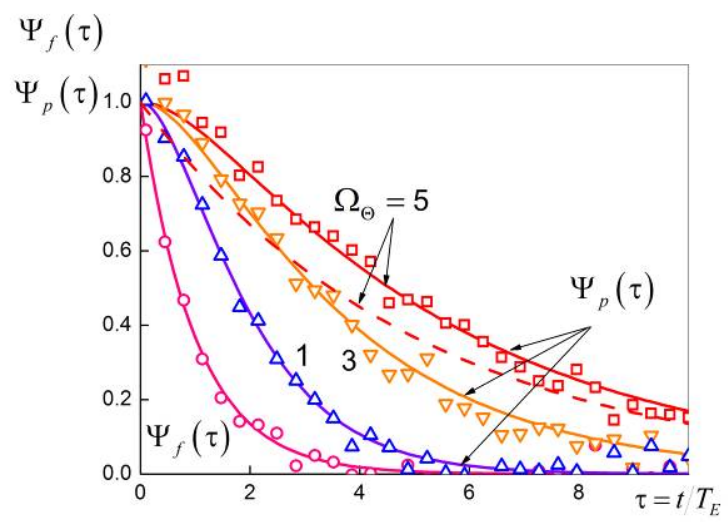

Fig. 5. Autocorrelation functions of temperature fluctuations fluid and granules. Points are numerical simulations, lines are the formulas (10) and (13).

\section{Simulation of THERMAL EXPLOSION. AVERAGE WAITING TIME OF EXPLOSION}

This section presents results showing the various scenarios of behavior of granules temperature of with internal heat generation in the fluid with fluctuating temperature. Figure 6 shows the behavior of the actual temperature of the granules with heat generation. On the figure $\Gamma_{f}=1+\gamma_{f}(t)$ is actual temperature of surrounding fluid. It can be seen that fluctuations in a magnitude of chemical reactions make a significant contribution to the value of the random temperature of granules. The initial temperature of the granule is less than the critical value $\Gamma_{I I}$ corresponding to the second root in the Semenov's diagram.

Random process with nonzero probability may exceed any level. After some random time the actual temperature of the granule will be over the critical value $\Gamma_{c r}=\Gamma_{I I}$ and there will be a loss of thermal stability. This scenario is illustrated by Figure 7 .

The waiting time of a thermal explosion we define as the average time of first crossing random temperature of granules critical level $\Gamma_{c r}$. Waiting time of thermal explosion $\tau_{c r}$ is function of initial temperature of the granule $\Gamma_{p}^{\circ}$ (Fig. 8).

As initial temperature approaches to the critical value, the average waiting time of thermal explosion dramatically reduced. The critical temperature $\Gamma_{c r}$ essentially depends on parameter of thermal inertia.

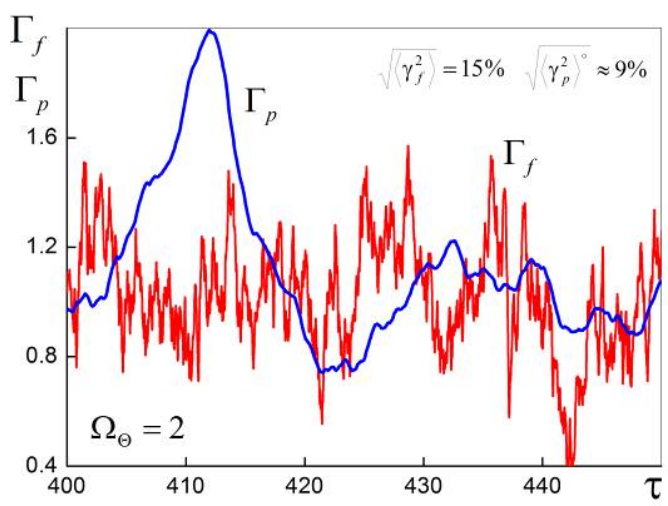

Fig. 6. No explosion.

\section{CONCLUSIONS}

Method of numerical simulation of random temperature of granules with internal heat source in surrounding liquid with 
temperature fluctuations is designed. The intensity of heat release is described by the Arrhenius law.

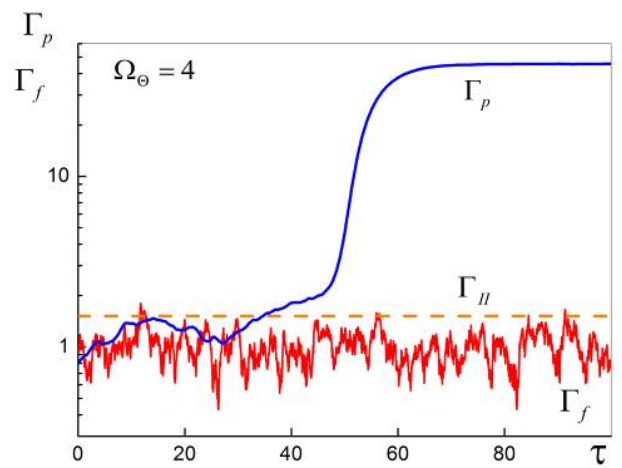

Fig. 7. Example of appearance of thermal explosion.

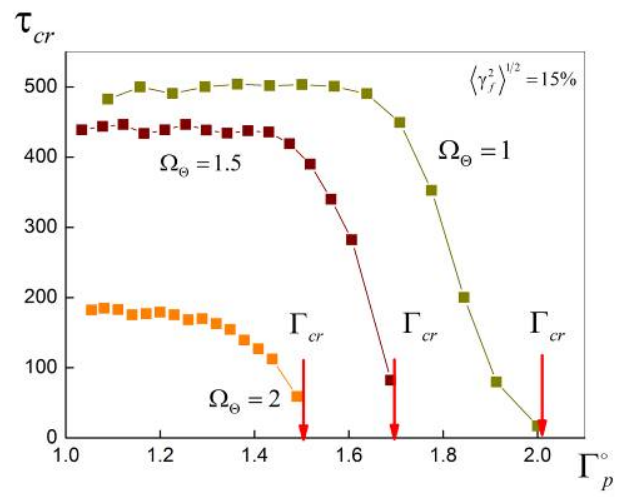

Fig. 8. Average waiting time of a thermal explosion.

For temperature fluctuations a numerical generation of random Gaussian process with an exponentially decaying autocorrelation function is suggested. Autocorrelation function and dispersions of temperature fluctuations without heat generation obtained by the numerical simulation are compared with the exact formulas, found by spectral analysis of stochastic processes.

Analysis of influence of the fluid temperature fluctuations on the process of thermal explosion is carried out. Dynamics of thermal explosion for various values of granules temperature relaxation times, initial temperature of granules, and dispersion of temperature fluctuations are investigated.

Based on direct numerical simulations the average waiting time of thermal explosion is investigated. Effect of stochastic drift of the granule temperature to its critical value is founded.

Further research in the area of numerical simulation is possible to carry out in two directions. Firstly, use of the actual kinetic schemes the Fischer-Tropsch synthesis on cobalt catalysts. The second direction of research focuses on the accounting of the random medium temperature intermittency characterized by the log-normal distribution.

\section{ACKNOWLEDGMENT}

This work was supported by the Russian Foundation for Basic Research (RFBR), grant number 11-08-00645-a.

\section{REFERENCES}

[1] A.P. Steynberg, M.E. Dry, B.H. Davis, B.B. Breman, "Chapter 2 Fischer-Tropsch Reactors", Studies in Surface Science and Catalysis, Vol. 152, pp. 64-195, 2004

[2] Ya.B. Zel'dovich, G.I. Barenblatt, V.B. Librovich, G.M. Makhviladze, Mathematical Theory of Combustion and Explosion, Nauka, Moscow, 1980 (in Russian).

[3] D.A. Frank-Kamenetskii, Diffusion and Heat Transfer in Chemical Kinetics, Plenum, New York, 1969.

[4] A.G. Merzhanov, E.N. Rumanov, "Nonlinear effects in macroscopic kinetics", Uspekhi Fizicheskikh Nauk, Vol. 151 (1987) 553-593 (in Russian).

[5] J. Warnatz, U. Maas, R.W. Dibble, Combustion. Physical and Chemical Fundamentals, Modeling and Simulations, Experiments, Pollutant Formation, Springer, 2001.

[6] W. Horsthemke, R. Lefever, Noise-induced Transitions. Theory and Applications in Physics, Chemistry and Biology, Springer, 1984.

[7] I. V. Derevich, R. S. Gromadskaya, "Rate of chemical reactions with regard to temperature fluctuations", Theoretical Foundations of Chemical Engineering, Vol. 31, No. 4, pp. 392-397, 1997.

[8] V.G. Medvedev, V.G. Telegin, G.G. Telegin, "Statistical analysis of kinetics of an adiabatic thermal explosion", Combustion, Explosion, and Shock Waves, Vol. 45, pp. 274-277, 2009.

[9] I.V. Derevich, "Temperature oscillation in a catalytic particle of Fischer-Tropsch synthesis", International Journal of Heat and Mass Transfer, Vol. 53, pp. 135-153, 2010.

[10] I.V. Derevich, "Effect of temperature fluctuations of fluid on thermal stability of particles with exothermic chemical reaction", International Journal of Heat and Mass Transfer, Vol. 53, pp. 5920-5932, 2010.

[11] I. V. Derevich, "Influence of temperature fluctuations on the thermal explosion of a single particle", Combustion, Explosion, and Shock Waves, Vol. 47, pp. 538-547, 2011.

[12] V.I. Klyatskin, Stochastic Equations Eyes of the Physicist: Substantive Provisions, Exact Results and Asymptotic Approaches, FIZMATHLIT, Moscow, 2001 (in Russian)

[13] G.Y. Liang, L. Cao, D.J. Wu, “Approximate Fokker-Planck equation of system driven by multiplicative colored noises with colored crosscorrelation", Physica A, Vol. 335, pp. 371 - 384, 2004.

[14] D. T. Gillespie, "Exact numerical simulation of the Ornstein-Uhlenbeck process and its integral", Physical Review E, Vol. 54, pp. 2084-2091, 1996.

[15] S. Ilie, A. Teslya, "An adaptive stepsize method for the chemical Langevin equation", Journal of Chemical Physics, Vol. 136, pp.184101 (14), 2012.

[16] C.W. Gardiner, Handbook of Stochastic Methods for Physics, Chemistry and the Natural Sciences, Springer, 1983.

[17] P. Lévy, Processus Stochastiques et Mouvement Brownien, GauthierVillars, Paris, 1965.

\section{Creative Commons Attribution License 4.0 (Attribution 4.0 International, CC BY 4.0)}

This article is published under the terms of the Creative Commons Attribution License 4.0

https://creativecommons.org/licenses/by/4.0/deed.en_US 